\title{
Incidence of pathogen detection in blood cultures of severe sepsis and septic shock patients is higher, if blood cultures were drawn before anti-infective therapy
}

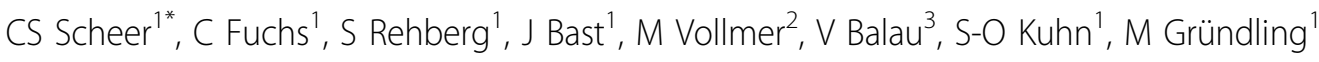 \\ From ESICM LIVES 2015 \\ Berlin, Germany. 3-7 October 2015
}

\section{Introduction}

Actual guidelines for the management of severe sepsis and septic shock recommend to draw blood cultures before anti-infective therapy. The grade of recommendation for this approach is high, the evidence, however, is low (1C) [1]. Thus, data on the influence of anti-infective therapy on microbiology results in seriously ill patients are urgently warranted.

\section{Objectives}

The objective of the present study was to describe the impact of a preexisting anti-infective therapy on the incidence of pathogen detection from blood cultures in patients with severe sepsis or septic shock.

\section{Methods}

Therefore, we retrospectively analyzed the results of 4498 blood cultures ( 2 bottles, each with 8-10 ml, BD BAC$\mathrm{TEC}^{\mathrm{TM}}$ aerobic/anaerobic medium bottles per drawn) from 592 intensive care unit (medical and surgical) patients with severe sepsis or septic shock from 2010 to 2014 at the University Hospital of Greifswald, Germany. The results were rated in consideration of anti-infective therapy, time of origin of severe sepsis and septic shock and time of blood culture drawing. Values were calculated as percentage and 95\%-confidence-intervals by Clopper-Pearson method.

\section{Results}

4210 blood cultures of 580 patients with severe sepsis or septic shock were included. 288 blood cultures of

${ }^{1}$ Universitätsmedizin Greifswald, Klinik für Anästhesiologie, Greifswald, Germany

Full list of author information is available at the end of the article
12 patients were excluded because of incomplete data. The patients were surgical and also medical with mostly abdominal (47.6\%) and pulmonary (26.4\%) focus. A median of 5 (interquartile range 3-10) blood cultures per patient were drawn. Overall 20.2\% (95\%CI 19.021.5) blood cultures were positive for pathogens. $18.4 \%$ $(774 / 4210)$ of blood cultures were drawn without and $81.6 \%(3436 / 4210)$ during anti-infective therapy. The positivity rate for all blood cultures without anti-infective therapy was $29.7 \%$ (95\%CI $26.5-33.1$ ) and $18.1 \%$ (95\%CI 16.8-19.4) during anti-infective therapy ( $\mathrm{p}<$ $0.001)$. Only $32.3 \%(1360 / 4210)$ were drawn within day 0 of origin of severe sepsis or septic shock and day 1 . In this period the positivity without anti-infective therapy was $33.8 \%$ (95\%CI $27.6-40.4)$ and $19.2 \%$ (95\%CI $17.0-$ $21.7)$ during anti-infective therapy $(\mathrm{p}<0.001)$. Figure 1 shows the differences in positivity rate at day 0 and 1 of severe sepsis or septic shock.

\section{Conclusions}

The present results support the current recommendation to draw blood cultures before anti-infective therapy. The highest probability of positive pathogen detection is at origin of severe sepsis or septic shock. During antiinfective the detection of pathogens is significantly decreased.

\section{Authors' details}

${ }^{1}$ Universitätsmedizin Greifswald, Klinik für Anästhesiologie, Greifswald, Germany. ${ }^{2}$ Ernst Moritz Arndt Universität Greifswald, Institut für Mathematik und Informatik, Greifswald, Germany. ${ }^{3}$ Universitätsmedizin Greifswald, Friedrich Loeffler Institut für Medizinische Mikrobiologie, Greifswald, Germany. 


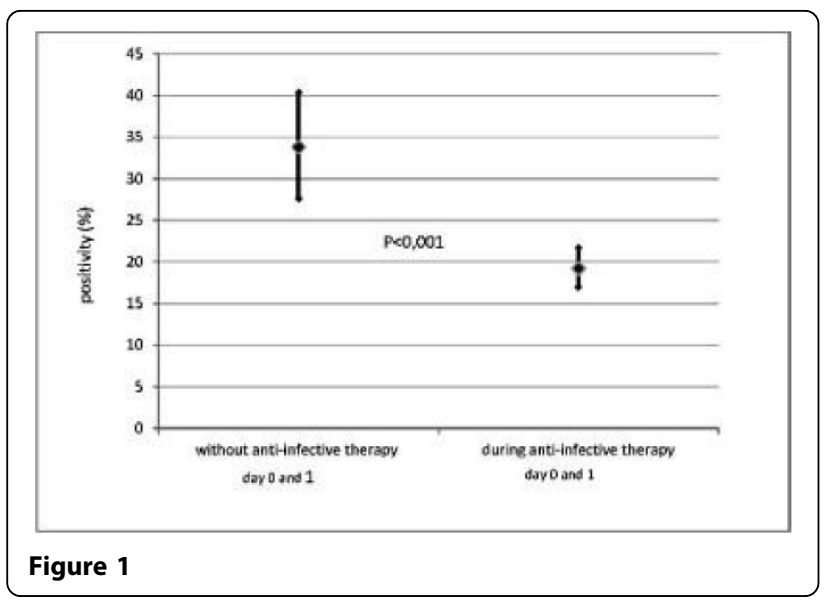

Published: 1 October 2015

\section{Reference}

1. Dellinger Crit Care Med 2013.

doi:10.1186/2197-425X-3-S1-A882

Cite this article as: Scheer et al.: Incidence of pathogen detection in blood cultures of severe sepsis and septic shock patients is higher, if blood cultures were drawn before anti-infective therapy. Intensive Care Medicine Experimental 2015 3(Suppl 1):A882.

\section{Submit your manuscript to a SpringerOpen ${ }^{\mathcal{O}}$ journal and benefit from:}

- Convenient online submission

- Rigorous peer review

- Immediate publication on acceptance

- Open access: articles freely available online

- High visibility within the field

- Retaining the copyright to your article

Submit your next manuscript at $\boldsymbol{\wedge}$ springeropen.com 